Klimakterisch und depressiv

\title{
Hormonersatztherapie trotz Mastopathie?
}

\author{
Frage: Ich betreue eine 52-jährige \\ Patientin, die seit Eintritt ins Klimakte- \\ rium vor acht Jahren an rezidivierenden \\ depressiven Zuständen leidet und \\ seither mit Venlafaxin behandelt wird. \\ Aufgrund einer fibrösen Mastopathie \\ wurde eine Hormonersatztherapie \\ (HRT) als kritisch eingestuft. Weil die \\ langfristige Wirkung von Venlafaxin als \\ ungenügend gesehen werden muss, \\ möchte die Patientin nun eine HRT \\ versuchen. Die körperlichen klimakteri- \\ schen Beschwerden sind auf gelegentli- \\ che Schweißausbrüche und trockene \\ Schleimhäute zurückgegangen. Ist eine \\ HRT möglich bzw. indiziert, und wenn \\ ja wie lange? Welches Präparat wäre zu \\ favorisieren?
}

Prof. Dr. Burkhard Schauf, Bamberg: Mastopathie ist keine Kontraindikation für eine HRT, sie kann sich sogar bessern. Die Ergebnisse zur Wirksamkeit einer HRT bei depressiven Zuständen sind kontrovers. Gesichert kann gelten, dass die HRT nicht negativ wirkt, falls es sich um endogene Depressionen handelt, und dass sie bei „depressiven Verstimmungen“ häufig positiv wirkt. Die Wirkung einer HRT auf trockene Schleimhäute und Schweißausbrüche ist natürlich sehr gut.

Ich würde zu einer kombinierten HRT mit androgenem Gestagen raten, wobei hier Kombipflaster günstig sind. Diese haben Norethisteronazetat (NETA) oder Levonorgestrel (LNG) als androgenes Gestagen und das Sexualhormon-bindende Globulin (SHBG) wird nicht stimuliert, d.h. Testosteron ist besser verfügbar, was in diesem Fall günstig ist.

Gut wäre auch Tibolon. Zu beachten ist dabei, dass es laut der LIBERATEStudie nach Brustkrebs problematisch ist, aber das Primärrisiko für Brustkrebs laut der LIFT-Studie eher senkt. Außerdem wirkt es aufgrund der starken androgenen Partialwirkung gut auf Mastopathie und Stimmung. Desweiteren sinkt SHBG stark ab, weshalb es in diesem Fall für mich eigentlich das Präparat der Wahl wäre.

Expertenrat Gynäkologie www.springermedizin.de

\section{Wem sollte man den Vorzug geben?}

\section{AT-Rezeptorblocker oder ACE-Hemmer?}

Frage: Ein Kollege erzählte mir, dass er wohl grundsätzlich keine ACE-Hemmer mehr einsetzen würde, wenn die Angiotensin-Rezeptorblocker (ARB) auf einem vergleichbaren Preisniveau lägen, was ja irgendwann zu erwarten ist. Teilen Sie grundsätzlich diese Ansicht? Aufgrund der Nebenwirkungen könnte man dazu kommen, wobei ich sehr viele Patienten sehe, die ohne jegliche unerwünschte Arzneimittelwirkungen jahrelang mit ihrem ACEHemmer auskommen. Warum sollte man da wechseln bzw. immer First-Line auf einen AT-Rezeptorblocker setzen?

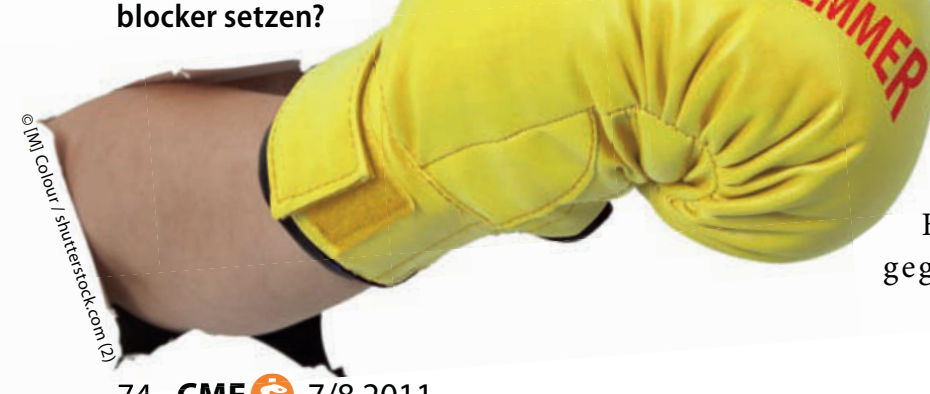

Prof. Dr. Matthias Girndt, Halle (Saale): Ich möchte auf die Ergebnisse der ONTARGET-Studie hinweisen, die als einzige große Studie einen ACE-Hemmer (Ramipril) gegen einen ARB (Telmisartan) bei jeweils mehr als 8000 Patienten mit Hypertonie und hohem kardiovaskulärem Risiko getestet hat. Bekanntlich zeigte sich keinerlei Unterschied zwischen beiden Substanzen. Sowohl für ACEHemmer als auch ARB gibt es theoretische Argumente, warum sie vorzuziehen seien. So blockieren ARB nicht die Effekte des Angiotensin-II-Rezeptors Typ 2, der Vasodilatation, Osmoregulation und Funktionen des Gefäßendothels beeinflusst. ACEHemmer hingegen hemmen den Bradykininabbau, was aufgrund der besseren Blutdrucksenkung günstig oder aufgrund von Husten oder einem Angioödem nachteilig sein kann. Von klinischer Relevanz scheinen diese Unterschiede allenfalls in Hinblick auf die Nebenwirkungen zu sein. Das einzige mir stichhaltig erscheinende Argument ist, dass bei schwerer Herzinsuffizienz ACE-unabhängig Angiotensin II gebildet werden kann, hier ein ARB also mehr Wirkungssicherheit bietet. Ansonsten halte ich beide Medikamentengruppen bei denen, die sie vertragen, für absolut gleichwertig.

Expertenrat Hypertonie \& Kreislauf www.springermedizin.de

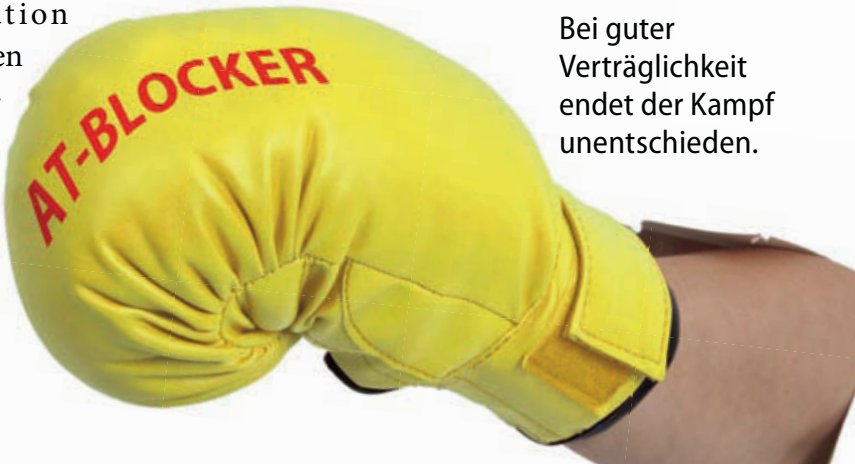

